\title{
Competition of Wireless Providers for Atomic Users: Equilibrium and Social Optimality
}

\author{
Vojislav Gajić *, Jianwei Huang ${ }^{\dagger}$, and Bixio Rimoldi * \\ ${ }^{*}$ School of Computer and Communication Sciences \\ ${ }^{\dagger}$ Department of Information Engineering \\ Ecole Polytechnique Fédérale de Lausanne \\ Lausanne, Switzerland \\ \{vojislav.gajic, bixio.rimoldi\}@epfl.ch \\ the Chinese University of Hong Kong \\ Shatin, Hong Kong \\ jwhuang@ie.cuhk.edu.hk
}

\begin{abstract}
We study a problem where wireless service providers compete for heterogenous and atomic (non-infinitesimal) wireless users. The users differ in their utility functions as well as in the perceived quality of service of individual providers. We model the interaction of an arbitrary number of providers and users as a two-stage multi-leader-follower game, and prove existence and uniqueness of the subgame perfect Nash equilibrium for a generic channel model and a wide class of users' utility functions. We show that, interestingly, the competition of resource providers leads to a globally optimal outcome under fairly general technical conditions. Our results show that some users need to purchase their resource from several providers at the equilibrium. While the number of such users is typically small (smaller than the number of providers), our simulations indicate that the percentage of cases where at least one undecided user exists can be significant.
\end{abstract}

\section{INTRODUCTION}

Due to the deregulation of telecommunication industry, one can imagine that in the future wireless users will not be contractually tied to a single service provider, but be free to switch in real time to the provider (or providers) offering the best tradeoffs. In this work, we consider a situation where wireless service providers want to earn profit by selling limited amount of wireless resources (e.g., frequency bands, time slots, transmission power) to a group of users. The users are rational economic agents who experience different channel conditions to the base stations of different providers and differ in willingness to pay.

The focus of our study is to understand the user-provider association and resource allocation in a general heterogenous wireless network setting. We model this interaction as a twostage extensive game of complete information. The providers announce resource prices in the first stage, and the users select which provider(s) to connect to as well as the corresponding amount of resource in the second stage. A user may choose a provider with an inferior channel if the price of the resource

This work is supported by the General Research Fund (Project Number 412308 and 412509) established under the University Grant Committee of the Hong Kong SAR, China, the National Key Technology R\&D Program (Project Number 2007BAH17B04) established by the Ministry of Science and Technology of China, and the Microsoft-CUHK Joint Laboratory for Humancentric Computing \& Interface Technologies. Part of the work was done when V. Gajic visited the Chinese University of Hong Kong in 2008. is low enough, or choose a more expensive provider if the channel is better. The providers select their prices to maximize their revenues, keeping in mind the impact of their prices on the demand of the users. As in [1], [2], we consider the case where users pay for the resources they use instead of the services they receive.

The contributions of our work are as follows:

- General Heterogeneous Wireless Network Model: We study a general network model that captures the heterogeneity of wireless users and service providers. The users have different utility functions, the providers have different resource constraints, the channel gains between users and providers are modeled by independent continuous random variables of arbitrary distributions (not necessarily and more general than location dependent), and the numbers of users and providers can be arbitrary.

- Unique Socially Optimal Allocation: We first study the problem of maximizing social welfare under the general utility function model. Despite the non-strict convexity of the optimization problem, we show that with probability 1 there exists a unique optimal solution to the problem. This follows from the fact that channel gains are drawn from continuous distributions so the event that multiple solutions exist has zero probability.

- Existence, Uniqueness, and Zero Efficiency Loss of Equilibrium: We further prove existence and uniqueness of the subgame perfect Nash equilibrium in the two-stage game. Moreover, we show that the unique equilibrium maximizes the social welfare under an easily verifiable sufficient condition on the users' utility functions, despite the selfish nature of the providers and users.

- Fractional Equilibrium with Atomic Users: Our model represents the case of finitely many atomic users, where each user has a finite demand so a single user's impact on the provider equilibrium can not be ignored. We show that the unique equilibrium of the game can possibly be fractional, where some users will purchase resource from two or more providers simultaneously. We provide an algorithm that uniquely determines such fractional equilibrium under the proper equilibrium prices. 
Linear usage-based pricing schemes are frequently considered in the literature, (e.g. [3]-[5]). Analyzing such pricing yields more than just economic insight: for example the existing TCP protocol can be interpreted as a usage-based pricing scheme that solves a network utility maximization problem [3]. Schemes where users pay for used resources and not obtained services often lead to desirable outcomes. In [1] and [6], users are charged for sent instead of received packets; as a result users reduce their transmission probabilities when congestion occurs. In a wireless setting, a usage based system gives users an incentive to chose primarily the provider that offers a good channel quality, which leads to more efficient resource utilization. The benefits of usage-based pricing schemes are clear despite some unresolved implementational issues (e.g., on the fly billing, global secure identities, etc.) [7], [8].

There exists a rich body of related literature on using pricing and game theory to study provider resource allocation and interactions of service providers. The related research in the wireless setting can be divided into several categories: optimization-based resource allocation of one provider (e.g., [9]-[13]), game theoretical interactions between the users of one provider (e.g., [14]-[16]), competition of different service providers on behalf of the users (e.g., [17], [18]), and providers' price competition to attract users (e.g., [19]-[26]). Our current work falls into the last category.

In our work we simultaneously consider several assumptions that reflect diverse wireless network scenarios: an arbitrary number of wireless providers compete for an arbitrary number of atomic users who are heterogenous in their channels gains and in their willingness to pay. In related work where providers price-compete to attract users [19], [21], [22], purchasing a unit of resource from different providers brings the same amount of utility to a user. In related results where Wardrop concept is used (e.g., [6], [24], [25]) users are infinitesimal and non-heterogenous. One of the early work that explicitly takes into account the channel differences for different (but non-atomic) users is [26]. Recently, a model similar to ours (although for less general utilites and channel gains) was considered for a three-tier system in [23].

As already mentioned, we assume that users are not only heterogenous in their utility functions, but also in terms of how they perceive the resource sold by the providers, which is a distinct feature of wireless communication. As a consequence of this model, even identical providers (in terms of available resource) are perceived differently by each user, leading to an asymmetric equilibrium with probability 1 .

After introducing the provider competition game, utility functions and communication model in Section II, we discuss the socially optimal resource allocation in Section III and the (subgame perfect) Nash equilibrium in Section IV. We provide some numerical results and dicussion in Section $\mathrm{V}$ and conclude in Section VI.

\section{PRoblem Formulation}

We consider a set $\mathcal{J}=\{1, \ldots, J\}$ of service providers and a set $\mathcal{I}=\{1, \ldots, I\}$ of users. Provider $j \in \mathcal{J}$ maximizes its revenue by selling a maximum amount of $Q_{j}$ resource to the users. A user $i \in \mathcal{I}$ maximizes its payoff by purchasing resources from one or more providers. We model the interaction as a multi-leader-follower game (see [27], [28]), where providers are the leaders and users are the followers. We assume that the channel gains of all users are roughly constant for the duration of the game, and furthermore, that they are known to all game participants (e.g. each provider collects its channel condition information to each user, and then broadcasts this information to all users and providers).

\section{A. Provider competition game}

Definition 1: (Provider competition game) The game consists of two stages. In the first stage, providers announce prices $\boldsymbol{p}=\left[p_{1} \cdots p_{J}\right]$, where $p_{j}$ is the unit resource price chosen by provider $j$. In the second stage, user $i \in \mathcal{I}$ chooses a demand vector $\boldsymbol{q}_{\boldsymbol{i}}=\left[\begin{array}{lll}q_{i 1} & \cdots & q_{i J}\end{array}\right]$, where $q_{i j}$ is the demand to provider $j$. We denote by $\boldsymbol{q}=\left[\boldsymbol{q}_{1} \cdots \boldsymbol{q}_{I}\right]$ the demand vector of all users. Taking prices $\boldsymbol{p}$ as given, a user $i$ 's goal is to choose $\boldsymbol{q}_{i}$ to maximize its payoff (i.e., utility minus payment):

$$
v_{i}\left(\boldsymbol{q}_{\boldsymbol{i}}, \boldsymbol{p}\right)=u_{i}\left(\sum_{j=1}^{J} q_{i j} c_{i j}\left(h_{i j}\right)\right)-\sum_{j=1}^{J} p_{j} q_{i j},
$$

where $h_{i j}$ is the channel gain between user $i$ and base station of provider $j$, and $c_{i j}(h)$ is the channel quality offset function. A provider $j$ chooses price $p_{j}$ to maximize its revenue $p_{j} \sum_{i=1}^{I} q_{i j}$ subject to the resource constraint $\sum_{i=1}^{I} q_{i j} \leq Q_{j}$ by taking into account the demand of the users in the second stage. Note that we consider linear pricing and no price discrimination across the users.

Under this model, a user is allowed to purchase from several providers at the same time, which gives an upper bound on efficiency of any situation where users purchase from one provider alone. Interestingly, our results show that for most users the optimal strategy is to choose exactly one provider.

\section{B. Model assumptions}

We make the following assumptions about the model:

Assumption 1: Utility functions: For every user $i \in \mathcal{I}, u_{i}(x)$ is differentiable, strictly increasing and strictly concave in $x$.

Assumption 2: Channel gains and channel quality offset functions: Channel gains $h_{i j}$ are drawn from arbitrary independent continuous probability distribution (e.g., Ralyleigh, Rician, distance-based path-loss model). The offset function $c_{i j}(h)$ is increasing and continuous in $h$. As a result, $c_{i j}$ 's also follow continuous distributions and $\operatorname{Pr}\left(c_{i j}=c_{k l}\right)=0$ for any $i, k \in \mathcal{I}, j, l \in \mathcal{J}$. The offset function accounts for the effect that buying the same amount of resource from different providers typically has different effects on a user's quality of service. For notation simplicity we write $c_{i j}$ instead of $c_{i j}\left(h_{i j}\right)$ and recall that $c_{i j}$ is increasing in $h_{i j}$.

Assumption 3: Atomic and price-taking users: We assume atomic users where the demand of each user is not infinitely small and can have an impact on providers' prices. Precise characterization of this impact is one of the focuses of this 
paper. On the other hand, users are price-takers by the assumption of the two-stage game, and can not strategically influence prices.

We illustrate the physical meaning of our model by the following example:

Example 1: Consider wireless providers operating on orthogonal frequency bands $W_{j}, j \in \mathcal{J}$, and let $q_{i j}$ be the fraction of time that user $i$ is allowed to transmit exclusively on the frequency band of network $j, \sum_{i \in \mathcal{I}_{j}} q_{i j}=1, j \in \mathcal{J}$. Furthermore, assume that each user has a peak power constraint $P_{i}$. Then, by taking $c_{i j}=W_{j} \log \left(1+\frac{P_{i}\left|h_{i j}\right|^{2}}{W_{j} \sigma_{i j}^{2}}\right)$, where $\sigma_{i j}^{2}$ is the noise variance of an additive white Gaussian noise (AWGN) channel between user $i$ and network $j$, a user's utility becomes an increasing function of obtained rate, with diminishing returns: $v_{i}=u_{i}\left(\sum_{j=1}^{J} q_{i j} c_{i j}\right)-\sum_{j=1}^{J} p_{j} q_{i j}$.

Similarly, our model can be used if providers are selling exclusive access to other types of resource (e.g., bandwidth, OFDM tones) with the peak power of a user fixed. In Example 1 , the $c_{i j}$ offset factor was made to be the channel capacity, but it may be any increasing function of the channel gain. In particular it may model any coding schemes or technology where rate is an increasing function of channel gain.

To analyze the properties of the provider competition game, in Section III we study a related socially optimal resource allocation problem and show the uniqueness of its solution (in terms of users' demands). Then, in Section IV, we show that such solution corresponds to the unique equilibrium of the game, in which case the selfish and strategic behavior of providers and users leads to zero efficiency loss.

\section{Social Optimum}

\section{A. Social welfare maximization}

In this Section we consider the social welfare problem, which aims at maximizing the summation of the users' payoffs and the providers' revenues. This is equivalent to maximizing the sum of users' utility functions since the payments between users and providers are system internal transfers and thus cancel out. For the following exposition, it will be useful to define $\boldsymbol{x}=\left[\begin{array}{lll}x_{1} & \cdots & x_{I}\end{array}\right]$ as the vector of weighted resources, where $x_{i}\left(\boldsymbol{q}_{i}\right)=\sum_{j=1}^{J} q_{i j} c_{i j}$.

The social welfare optimization problem NUM (network utility maximization) is:

$$
\begin{aligned}
\max u(\boldsymbol{x})= & \sum_{i=1}^{I} u_{i}\left(x_{i}\right) \\
\text { subject to } & \sum_{j=1}^{J} q_{i j} c_{i j}=x_{i} i \in \mathcal{I} \\
& \sum_{i=1}^{I} q_{i j}=Q_{j}, j \in \mathcal{J} \\
\text { over } & q_{i j} \geq 0 \forall i \in \mathcal{I}, j \in \mathcal{J} .
\end{aligned}
$$

There are two different variables in NUM: weighted resource vector $\boldsymbol{x}$ and demand vector $\boldsymbol{q}$. In particular, a vector $\boldsymbol{q}$ uniquely determines a vector $\boldsymbol{x}$ through equations (3) (we write $x(\boldsymbol{q})=\boldsymbol{x})$, which in term uniquely determines the objective function $u(\boldsymbol{x})$. With some abuse of notation we will write $u(\boldsymbol{q})$ when we mean $u(x(\boldsymbol{q}))$.

Lemma 1: The social welfare optimization problem NUM has a unique maximizing solution $\boldsymbol{x}^{*}$.

Proof: Since $u_{i}\left(x_{i}\right)$ is strictly concave in $x_{i}$, then $u(\boldsymbol{x})=$ $\sum_{i=1}^{I} u_{i}\left(x_{i}\right)$ is strictly concave in $\boldsymbol{x}$. The feasible region defined by constraints (3)-(5) is convex. Hence, $u(\boldsymbol{x})$ has a unique maximizing solution $\boldsymbol{x}^{*}$.

\section{B. Uniqueness of the socially optimal demand vector $\boldsymbol{q}^{*}$}

Even though $u_{i}(\cdot)$ 's are strictly concave in $x_{i}$, they are not strictly concave in the demand vector $\boldsymbol{q}_{\boldsymbol{i}}$. Hence, NUM is non-strictly concave in $\boldsymbol{q}$. It is well-known that a non-strictly concave maximization problem might have several different global optimal optimizers (several different demand vectors $\boldsymbol{q}$ in our case) (see e.g. [29], [30]). In particular, one can choose $c_{i j}$ 's, $Q_{j}$ 's and $u_{i}(\cdot)$ 's in such a way that a maximizing vector of NUM is not unique. However, it turns out that such cases arise with zero probability whenever channel offsets factors $c_{i j}$ 's are drawn from continuous distributions.

In the remainder of this Section we show that NUM has a unique maximizing demand vector with probability 1 . We begin by proving Lemma 2, an intermediary result stating that any two maximizing demand vectors of NUM must have different non-zero components. We observe that any convex combination of two maximizing demand vectors is also a maximizing demand vector. We then show that all convex combinations of maximizing demand vectors have the same non-zero components, which is a contradiction with Lemma 2. This proves the main result of this Section (Theorem 3).

To make our argument precise, let us first define the support set of a demand vector $\boldsymbol{q}_{i}$ as follows.

Definition 2: (Support set): The support set of $\boldsymbol{q}_{i}$ contains the indices of its non-zero entries, i.e.:

$$
\hat{\mathcal{J}}_{i}\left(\boldsymbol{q}_{i}\right)=\left\{j \in \mathcal{J}: q_{i j}>0\right\} .
$$

Given a demand vector $\boldsymbol{q}$, the ordered collection of support sets $\hat{\mathcal{J}}_{1}, \hat{\mathcal{J}}_{2}, \ldots, \hat{\mathcal{J}}_{I}$ is denoted by $\left\{\hat{\mathcal{J}}_{i}\right\}_{i=1}^{I}$.

The support set $\mathcal{J}_{i}$ contains the providers that user has strictly positive demand from.

Lemma 2: Let $\left\{\hat{\mathcal{J}}_{i}\right\}_{i=1}^{I}$ be a collection of support sets of some maximizing demand vector $\boldsymbol{q}^{*}$. Then, $\boldsymbol{q}^{*}$ is almost surely ${ }^{1}$ the unique maximizing demand vector with that specific collection of support sets.

Proof: Consider a collection of support sets $\left\{\hat{\mathcal{J}}_{i}\right\}_{i=1}^{I}$ constructed from some maximizing demand vector $q^{*}$ (a maximizing demand vector satisfies $x\left(\boldsymbol{q}^{*}\right)=\boldsymbol{x}^{*}$ and equations (4) and (5)). To prove the lemma, we will uniquely construct $\boldsymbol{q}^{*}$ from $\boldsymbol{x}^{*}$ and $\left\{\hat{\mathcal{J}}_{i}\right\}_{i=1}^{I}$.

The users can be divided into three categories. The decided users purchase from only one provider $\left(\left|\hat{\mathcal{J}}_{i}\right|=1\right)$, and the undecided users from several $\left(\left|\hat{\mathcal{J}}_{i}\right|>1\right)$. It is also possible that some users have zero demand to all providers, i.e. for such

\footnotetext{
${ }^{1}$ This holds on the probability space defined by the distributions of $c_{i j}$ 's.
} 
users $\boldsymbol{q}_{i}^{*}=\mathbf{0}$, the all zero vector. Without loss of generality, we treat such users as decided. For all users the demand vector $\boldsymbol{q}_{i}^{*}$ is such that $x_{i}^{*}=\sum_{j=1}^{J} q_{i j}^{*} c_{i j}$. For a decided user $i$ who purchases only from provider $\bar{j}$, this reduces to $x_{i}^{*}=q_{i \bar{j}}^{*} c_{i \bar{j}}$, so we can uniquely find the maximizing demand vector of a decided user $i$ as $\boldsymbol{q}_{i}^{*}=\left[0 \cdots 0 \frac{x_{i}^{*}}{c_{i \bar{j}}} 0 \cdots 0\right]$.

For undecided users, $\boldsymbol{q}_{i}^{*}$ cannot be found directly as there is more than one $\boldsymbol{q}_{i}^{*}$ such that $\sum_{j \in \hat{\mathcal{J}}_{i}} q_{i j}^{*} c_{i j}=x_{i}^{*}$. To show that the demand of undecided users is unique we use bipartite graphs. We construct the bipartite graph representation (BGR) $\mathcal{G}$ of the undecided users' support sets in the following way:

\begin{tabular}{l}
\hline BGR construction \\
1: Represent undecided users by circles. \\
2: Represent providers with undecided users as squares. \\
3: Place an edge $(i, j)$ between a provider node $j$ and a \\
user node $i$ if $j \in \hat{\mathcal{J}}_{i}$.
\end{tabular}

In Figure 1 we give an example of a BGR constructed for $\hat{\mathcal{J}}_{1}=\{a, b, c\}, \hat{\mathcal{J}}_{2}=\{b, d\}, \hat{\mathcal{J}}_{3}=\{d, e, f\}$ and $\hat{\mathcal{J}}_{4}=\{b, g\}$.

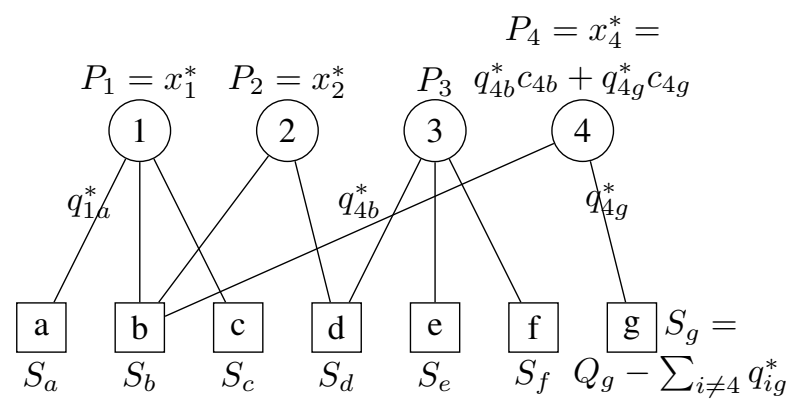

Fig. 1. Bipartite graph representation

The BGR has the following properties (refer to Figure 1):

1) A BGR is not necessarily a connected graph, i.e., it may consist of several components.

2) The sum of weighted resource on all the edges connecting user $i$ is the optimal weighted resource $x_{i}^{*}=$ $\sum_{j \in \hat{\mathcal{J}}_{i}} q_{i j}^{*} c_{i j}=P_{i}$. We call $P_{i}$ the check-sum of a user node.

3) The sum of all edges connecting to provider node $j$ is the supply $Q_{j}$ minus the demand from decided users who connect to provider $j: \sum_{i:(i, j) \in \mathcal{G}} q_{i j}^{*}=Q_{j}-$ $\sum_{i:(i, j) \notin \mathcal{G}} q_{i j}^{*}=S_{j}$. We call $S_{j}$ the check-sum of a provider node.

4) With probability 1 , the BGR does not contain any loops.

Property 4) is proved in Appendix A. Properties 1)-3) follow directly from the way that a BGR is constructed.

We now give an informal description of an algorithm that finds the optimal and unique values of $\boldsymbol{q}_{i}^{*}$ for undecided users (the formal description is given in [31]). We call this algorithm the BGR decoding algorithm. Since BGR has no loops, it is a (unrooted) tree. Hence, we can run a simple iterative algorithm which removes a leaf node (node with a single incoming edge) and its associated edge at each iteration. We begin by finding a leaf node in the BGR. We then determine the demand of the edge associated to the leaf node either from BGR Property 2) or 3 ). Using this value we update the check-sum value of the parent node. In the final step of the iteration we remove the leaf node and the associated edge. We then look for the next leaf node in the reduced graph and repeat the process: we remove the edge associated to the leaf node after determining the edge demand, find the next leaf node etc. We repeat the process until there are no more edges in the graph. Property 4) is crucial in this procedure since it guarantees that we can always find a leaf node in the reduced tree. Note that this property is a consequence of the model, and not an assumption.

We illustrate the procedure for determining $\boldsymbol{q}_{i}^{*}$ for undecided users using the example in Figure 1. Consider leaf node $g$ and edge $q_{4 g}^{*}$. This BGR implies that user 4 is the only undecided customer of provider $g$ (i.e. $q_{1 g}^{*}=q_{2 g}^{*}=q_{3 g}^{*}=0$ ), and since the demand of all decided customers was determined, it must be that $q_{4 g}^{*}=S_{g}=Q_{g}-\sum_{i \neq 4} q_{i g}^{*}$. We then remove edge $q_{4 g}^{*}$ and node $g$, and update the check-sum value of node 4 to $P_{4}=x_{4}^{*}-q_{4 g}^{*} c_{4 g}$. We can now consider node 4 and edge $q_{4 b}^{*}$. It must be that $q_{4 b}^{*} c_{4 g}=P_{4}$, hence $q_{4 b}^{*}=\frac{P_{4}}{c_{4 b}}$. Next we can consider node $a, e$, or $f$, and so on.

In each step of the algorithm we determine the unique value of $q_{i j}^{*}$ associated with an edge, and this is independent of the order in which we pick the leaf nodes. So, we can construct unique demand vectors $\boldsymbol{q}_{i}^{*}$ for all undecided users $i$ in the BGR. Hence, the constructed demand vector $\boldsymbol{q}^{*}$ is the only maximizing demand vector of NUM with collection of support sets $\left\{\hat{\mathcal{J}}_{i}\right\}_{i=1}^{I}$.

Theorem 3: The social welfare optimization problem NUM has a unique maximizing solution $\boldsymbol{q}^{*}$ with probability 1 .

Proof: Assume there exist two maximizing demand vectors $\boldsymbol{q}^{*}$ and $\boldsymbol{q}^{\prime}$. Then, by Lemma $2, \boldsymbol{q}^{*}$ and $\boldsymbol{q}^{\prime}$ have different collections of support sets $\left\{\hat{\mathcal{J}}_{i}^{*}\right\}_{i=1}^{I}$ and $\left\{\hat{\mathcal{J}}_{i}^{\prime}\right\}_{i=1}^{I}$ almost surely. Next, consider a convex combination demand vector $\boldsymbol{q}^{\lambda}=\lambda \boldsymbol{q}^{*}+\bar{\lambda} \boldsymbol{q}^{\prime}$ where $\lambda \in(0,1), \bar{\lambda}=1-\lambda$. Since $x_{i}^{*}=\sum_{j=1}^{J} q_{i j}^{*} c_{i j}=\sum_{j=1}^{J} q_{i j}^{\prime} c_{i j}$, then $\sum_{j=1}^{J} q_{i j}^{\lambda} c_{i j}=$ $\lambda \sum_{j=1}^{J} q_{i j}^{*} c_{i j}+\bar{\lambda} \sum_{j=1}^{J} q_{i j}^{\prime} c_{i j}=x_{i}^{*}$, so it follows that $\boldsymbol{q}^{\lambda}$ is also a maximizing solution of NUM for any $\lambda \in(0,1)$. Then, the support set $\hat{\mathcal{J}}_{i}^{\lambda}\left(\boldsymbol{q}^{\lambda}\right)=\left\{j \in \mathcal{J}: q_{i j}^{\lambda}=\lambda q_{i j}^{*}+\bar{\lambda} q_{i j}^{\prime}>0\right\}$ for user $i$ is $\hat{\mathcal{J}}_{i}^{\lambda}=\hat{\mathcal{J}}_{i}^{*} \cup \hat{\mathcal{J}}_{i}^{\prime}$, for all $\lambda \in(0,1)$. In particular, the support set collections $\left\{\hat{\mathcal{J}}_{i}^{\lambda}\right\}_{i=1}^{I}$ are the same for all $\lambda \in(0,1)$, which, using Lemma 2, implies that $\boldsymbol{q}^{\lambda}=\boldsymbol{q}$ for all $\lambda \in(0,1)$, which further implies $\boldsymbol{q}^{*}=\boldsymbol{q}^{\prime}=\boldsymbol{q}$.

Hence, the maximizing demand vector $\boldsymbol{q}^{*}$ of the social welfare optimization problem NUM is unique with probability 1. From optimization theory [32] we know that there will be a unique Lagrangue multiplier vector $p^{*}$ (with $J$ components) associated with the resource constraints of all providers of the unique optimal demand vector $q^{*}$. In the next section we show that $\left(\boldsymbol{q}^{*}, \boldsymbol{p}^{*}\right)$ is the unique equilibrium of the two-stage provider competition game. 


\section{AnAlysis of THE TWO STAGE GAME}

In this section we show that there exists a unique equilibrium (defined more precisely shortly) of the provider competition game. In particular, this equilibrium corresponds to the unique social optimal solution of the welfare optimization problem NUM and the associated Lagrange multipliers. The idea is to interpret these Lagrange multipliers as the prices proposed by the providers to the users. Moreover, we show that there are at most $J-1$ undecided users at this equilibrium.

As the equilibrium concept, we adopt the following definition of the subgame perfect equilibrium [33]:

Definition 3: (Subgame perfect equilibrium (SPE)) A price demand tuple $\left(\boldsymbol{p}^{*}, \boldsymbol{q}^{*}\left(\boldsymbol{p}^{*}\right)\right)$ is a subgame perfect equilibrium of the provider competition game if no player has an incentive to deviate unilaterally. In particular, each user $i \in \mathcal{I}$ maximizes its payoff given prices $\boldsymbol{p}^{*}$. Each provider $j \in \mathcal{J}$ maximizes its revenue given other providers' prices $p_{-j}^{*}=\left(p_{1}^{*}, \cdots, p_{j-1}^{*}, p_{j+1}^{*}, \cdots p_{J}^{*}\right)$ and the users' demand function $\boldsymbol{q}^{*}\left(\boldsymbol{p}^{*}\right)$.

\section{A. Equilibrium strategy of the users}

Notice that here we consider a perfect information game where each user/provider knows the complete information of other participants. Consider users who are facing prices $\boldsymbol{p}$ in the second stage. Each user is maximizing his payoff $u_{i}\left(\sum_{j=1}^{J} q_{i j} c_{i j}\right)-\sum_{j=1}^{J} p_{j} q_{i j}$. By differentiating with respect to $q_{i j}$ and examining the first order conditions, we see that the maximizing demand for user $i$ satisfies

$$
\frac{\partial u_{i}\left(x_{i}\right)}{\partial x_{i}}=\underset{k \in \mathcal{J}}{\operatorname{argmin}} \frac{p_{k}}{c_{i k}}, \text { where } x_{i}=\sum_{j \in \operatorname{argmin}_{k} \frac{p_{k}}{c_{i k}}} q_{i j} c_{i j},
$$

and $q_{i j}=0$ for $j \notin \operatorname{argmin}_{k \in \mathcal{J}} \frac{p_{k}}{c_{i k}}$. This motivates the following definition:

Definition 4: (Preference set) For any price vector $\boldsymbol{p}$, user $i$ 's preference set $\mathcal{J}_{i}(\boldsymbol{p})$ is the set of those providers $j \in \mathcal{J}$ with $\frac{p_{j}}{c_{i j}}=\min _{k \in \mathcal{J}} \frac{p_{k}}{c_{i k}}$. A collection of preference sets of all users $\mathcal{J}_{1}, \mathcal{J}_{2}, \ldots, \mathcal{J}_{I}$ is denoted by $\left\{\mathcal{J}_{i}\right\}_{i=1}^{I}$.

Since $u_{i}(\cdot)$ is strictly increasing and strictly concave, we see from (6) that a user's payoff is maximized by a unique value of $x_{i}$, which we denote $x_{i}^{*}$. Suppose that $x_{i}^{*}>0$ (otherwise $\boldsymbol{q}_{i}=0$ ). In this case we have again decided and undecided users as in the social welfare optimization problem in Section 3 . Here a decided user has a preference set with cardinality one, and there is a unique demand vector $\boldsymbol{q}_{i}$ (with only one positive element) that maximizes its payoff. For an undecided user, the cardinality of its preference set is larger than one, and this user has infinite choices of demand vectors to maximize its payoff (i.e. any $\boldsymbol{q}_{i} \geq \mathbf{0}$ such that $x_{i}^{*}=\sum_{j \in \mathcal{J}_{i}} q_{i j} c_{i j}$ ).

There is a close relationship between the support sets from Section III and preference sets. Namely, for any provider $j$ that the user $i$ gets positive resource from (i.e. $j \in \hat{\mathcal{J}}_{i}$ ) we have that $j$ is in the preferred set of that user $\left(j \in \mathcal{J}_{i}\right)$. Hence the support set of a user is a subset of its preference set: $\hat{\mathcal{J}}_{i}(\boldsymbol{q}(\boldsymbol{p})) \subset \mathcal{J}_{i}(\boldsymbol{p})$. In addition, we can construct a BGR based on the preference sets. It can be shown that, with probability
1, this BGR also has no loops (proof is similar to that of Appendix A).

In particular, since the provider competition game is a game of complete information, once the prices $\boldsymbol{p}$ are announced each user knows all other users' preference sets, and can construct the corresponding BGR. Undecided users can now uniquely determine their demand vector by running the BGR decoding algorithm. This is one of undecided user's infinitely many best responses. If all undecided users choose their resource amounts by running the BGR decoding algorithm with the same complete information, then the total demand to each provider equals his supply. However, if some of the users choose a different best response, total demand is not equal to supply for some providers who then have an incentive to change their strategy.

Hence, the only best response $\boldsymbol{q}^{*}(\boldsymbol{p})$ of the users that guarantees that they get what they ask for is to deduce their demand vectors from the BGR. More importantly, the providers know that BGR decoding algorithm is the best response of the users, so they can choose the prices such that the best response of the users leads to the maximized profit. We can also show that it is possible to achieve this best response by running a distributed algorithm that relies only on local information [34].

\section{B. Equilibrium strategy of the providers}

For a specific class of utility functions, it is easier to characterize the profit maximizing criterion for the providers. Let us define the coefficient of relative risk aversion of user $i$ as $k_{R R A}^{i}=-\frac{x u_{i}^{\prime \prime}(x)}{u_{i}^{\prime}(x)}$. The coefficient of relative risk aversion indicates a user's elasticity of demand.

Assumption 4: For each user $i \in \mathcal{I}$, the coefficient of relative risk aversion is less than 1 .

Assumption 4 implies that the demand of the users is such that a monopolistic provider should sell all of its resource $Q_{j}$ to maximize its revenue. It is satisfied by some commonly used utility functions (such as $\log (1+x)$ and the $\alpha$-fairness ${ }^{2}$ $\frac{x^{1-\alpha}}{1-\alpha}$, for $\alpha \in(0,1)$ [35], [36]). Based on this assumption, the providers maximize their profit when demand equals the supply.

\section{Analysis of the provider competition game}

Theorem 4: Under assumption 4, the unique socially optimal demand vector $\boldsymbol{q}^{*}$ and the associated Lagrangian multiplier vector $\boldsymbol{p}^{*}$ constitute the unique sub-game perfect equilibrium (SPE) of the provider competition game.

The proof is given in Appendix B.

It is quite surprising to see that the SPE of the provider competition game is also a social optimum, i.e. that the competition of providers does not reduce efficiency. This is not a simple consequence of the strict concavity of the utility functions of the users; instead it is also related to the elasticity of users' demand. If the users have utility functions that satisfy Assumption 4, then the best strategy for the providers is

\footnotetext{
${ }^{2}$ For $\alpha \geq 1$ the $\alpha$-fair utility function does not satisfy Assumption 4, where for $\alpha=1$ the $\alpha$-fair utility function is $\log (x)$.
} 
to sell all of their resource. Otherwise, the demand of the users may not be elastic enough, and a provider's strategy to maximize profit may be to increase the price beyond the market clearing price. For example, the provider competition game involving users with $\alpha$-fair functions with $\alpha>1$ will not have the socially optimal equilibrium since the profit maximizing strategy of a provider is to sell less than its entire resource ${ }^{3}$. The class of utility function resulting in a unique SPE (constrained by Assumption 4) is smaller than the class of utility functions resulting in a unique socially optimal allocation (Assumption 1).

What makes our analysis non-straightforward is the presence of undecided users, who are fundamentally different than decided users. To compute the equilibrium achieving best response, the undecided users need to take into account the information of other participants. This increases the complexity of decision making, so an interesting question that one might ask is how many undecided users can there be in a given game. Although in practice the number of users is generally much larger than the number of providers, it turns out that for any given price vector (and in particular the equilibrium price vector) there exist at most $J-1$ users who are undecided.

Lemma 5: Given any price vector $\boldsymbol{p}$, the number of undecided users is at most $J-1$.

The proof is omitted for brevity, and can be found in [31]. The main idea is to observe that if the number of undecided user nodes in a BGR is greater than the number of provider nodes (which is at most equal to the total number of providers), this leads to a BGR with loops, which by Property 4 occurs with zero probability.

\section{Simulations AND Discussion}

One of the result of our analysis is that some users may connect to more than one provider at the equilibrium. While Lemma 5 gives an upper bound to the number of undecided users, it does not say much about the possibility of having such users in the system. Our simulations results indicate that for a relatively large number of users a significant percentage of cases involves at least one undecided users. As an example, we consider a three provider network with the base-stations of the networks fixed, and users locations are drawn uniformly at random on a square area. Figure 2 shows that percentage of instantiations having undecided users at the equilibrium for different number of users (ranging from 10 to 50). We assume that three providers have equal total resource, i.e., $Q_{1}=Q_{2}=Q_{3}$. The users' utility function is $a_{i} \log \left(1+\sum_{j=1}^{3} q_{i j} c_{i j}\right)$, where $c_{i j}$ is the channel capacity $\log \left(1+\mathrm{SNR}\left|h_{i j}\right|^{2}\right), q_{i j}$ is the amount of allocated time, and $a_{i}$ 's are the individual willingness to pay factors (drawn from an exponential distribution). The channel gain amplitudes $\left|h_{i j}\right|$ are drawn from an exponential distribution with mean equal to the distance between user $i$ and provider $j$. For each choice of user number ranging from 10 to 50 , we generated 500 sets

\footnotetext{
${ }^{3}$ For users with $\log (x)$ utility function (i.e. $\alpha$-fair utility function with $\alpha=1$ ) the provider gets the same profit regardless of how much resource it sells to the user.
}

of channel coefficients, and found the percentage of equilibria with users connecting to multiple networks. The percentage has a decreasing trend with a larger user population, although it is significant even with 50 users.

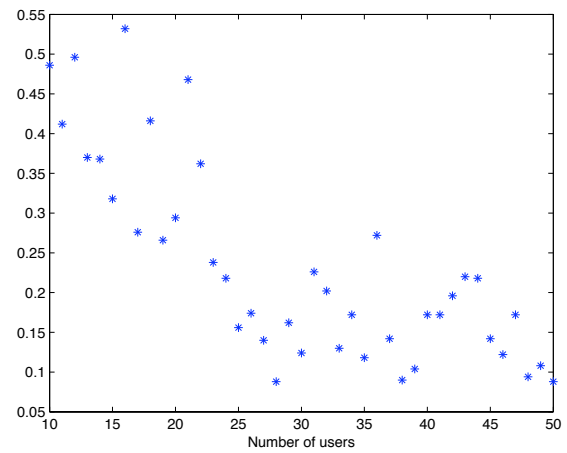

Fig. 2. Percentage of equilibria with at least one undecided user

Technically speaking, the difference between decided and undecided users is significant. The decided users have a unique demand vector that maximizes their payoffs, while the undecided users have an infinite number of such vectors. This is not an issue in the complete information case as we have described. However, in practical networks where full information is difficult to obtain, it would be interesting to know what kind of distributed learning algorithm can lead to the same unique equilibrium. We are investigating this as part of our ongoing work [34].

The number of undecided users is small and its upper bound $(J-1)$ does not grow with the number of users. Future systems may replace user choices by the actions of software agents in charge of connection and handover between different providers. In this case, splitting over different providers may become feasible. This is not very much unlike soft handoff (soft handover), a feature used by CDMA and WCDMA standards [37]. On the other hand, when the number of users is large, the impact on the price of a single undecided user may be small. Hence, sub-optimal decisions of a few undecided users may not have a great impact on the experienced quality of service, although the exact loss remains to be quantified.

In the model we consider, we observe the "locally monopolistic" nature of wireless commerce, which does not exist for most other traditional goods. Namely, a user that has a strong channel to some provider, but a weak one to others, is willing to pay a much higher price to the provider with the strong channel, and is thus not influenced by price competition. On the other hand, users with similar channel gains to all providers will be more sensitive to price competition. This local monopoly is in contrast to some other wireless resource allocation models where users' association is based solely on the price, so given the choice between wireless providers, all users go to the cheaper one. 


\section{Vi. CONClusions AND Future Work}

We have studied the competition of an arbitrary number of wireless service providers for atomic users who are heterogenous in both willingness to pay and the channel quality. We model this interaction as a two-stage wireless provider game and characterize its equilibrium state. We obtain a result that competition leads to the unique globally optimal outcome for a broad class of utility functions and a generic channel model. Our results show that some users need to purchase their resource from several providers at the equilibrium. While the number of such users is typically small (no larger than J-1), our simulations indicate that the percentage of cases where at least one undecided user exists can be significant. Our future work will focus on developing decentralized algorithms that result in equilibrium prices as well as the equilibrium demand vectors using only local knowledge. We will further study fractional equilibria with the goal of characterizing the losses that occur when undecided users are unable to split their resource demand in an optimal way. Finally, it would be interesting to consider communication models where users cause externalities (such as interference or congestion) to each other.

\section{APPENDIX A}

\section{PROOF OF BGR PROPERTY 4}

First, we look at the properties of the demand vector that maximizes NUM. We will express the NUM in terms of the demand vector $\boldsymbol{q}$ by substituting directly equation (3) into equation (2). Let $\boldsymbol{p}=\left[p_{1} \cdots p_{J}\right]$ be the vector of Lagrangian multipliers. The Lagrangian for NUM is then

$$
L(\boldsymbol{q}, \boldsymbol{p})=\sum_{i=1}^{I} u_{i}\left(\sum_{j=1}^{J} q_{i j} c_{i j}\right)+\sum_{j=1}^{J} p_{j}\left(Q_{j}-\sum_{i=1}^{I} q_{i j}\right) \text {. }
$$

Differentiating the Lagrangian with respect to $q_{i j}$ gives

$$
\frac{\partial L}{\partial q_{i j}}=\frac{\partial v_{i}\left(\sum_{j=1}^{J} q_{i j} c_{i j}\right)}{\partial q_{i j}}=\frac{\partial u_{i}\left(x_{i}\right)}{\partial x_{i}} c_{i j}-p_{j},
$$

where with some abuse of notation we write $\frac{\partial u_{i}\left(x_{i}\right)}{\partial x_{i}}$ instead of $\left.\frac{\partial u_{i}(x)}{\partial x}\right|_{x=x_{i}=\sum_{j=1}^{J} q_{i j} c_{i j}}$. The Karush-Kuhn-Tucker (KKT) conditions, i.e. the necessary and sufficient conditions fulfilled by the maximizing solution $\boldsymbol{q}$ for some $\boldsymbol{p}$ can be summarized as follows:

$$
\begin{aligned}
\frac{\partial u_{i}\left(x_{i}\right)}{\partial x_{i}} c_{i j}-p_{j} & \leq 0, j \in \mathcal{J} ; i \in \mathcal{I} \\
q_{i j}\left(\frac{\partial u_{i}\left(x_{i}\right)}{\partial x_{i}} c_{i j}-p_{j}\right) & =0, j \in \mathcal{J} ; i \in \mathcal{I} \\
\sum_{j=1}^{J} q_{i j} c_{i j} & =x_{i}, i \in \mathcal{I} \\
\sum_{i=1}^{I} q_{i j} & =Q_{j}, j \in \mathcal{J} \\
p_{j}>0, q_{i j} & \geq 0 j \in \mathcal{J} ; i \in \mathcal{I}
\end{aligned}
$$

We refer to equations (9)-(13) collectively as the KKT conditions, and say that any maximizing solution $\boldsymbol{q}$ must satisfy the KKT conditions for some Lagrange multiplier vector $\boldsymbol{p}$. Furthermore, any vector $\boldsymbol{q}$ that satisfies the KKT conditions for some $p$ is a maximizing vector of NUM. Using the Lagrange multipliers, let us define $\mathcal{J}_{i}(\boldsymbol{p})=\left\{j \in \mathcal{J}: \frac{p_{j}}{c_{i j}}=\right.$ $\left.\min _{k \in \mathcal{J}} \frac{p_{k}}{c_{i k}}\right\}$ for all $i \in \mathcal{I}$ (in Section IV this is the definition of the preference set of user $i$ given prices $p$, while here the sets are defined based on Lagrange multipliers). The following gives us a useful characterization for any two networks that a user has strictly positive demand from.

Recall that $\hat{\mathcal{J}}_{i}\left(\boldsymbol{q}_{i}\right)=\left\{j \in \mathcal{J}: q_{i j}>0\right\}$. From equation (10) we can see that $q_{i j}>0$ only when $\frac{\partial u_{i}\left(x_{i}\right)}{\partial x_{i}}=\frac{p_{j}}{c_{i j}}$. Hence $\frac{p_{j}}{c_{i j}}=\min _{k \in \mathcal{J}} \frac{p_{k}}{c_{i k}}$ is a necessary condition for $q_{i j}>0$, i.e. $\hat{\mathcal{J}}_{i}(\boldsymbol{q}) \subset \mathcal{J}_{i}(\boldsymbol{p})$, for all $i \in \mathcal{I}$. Then, $q_{i j}>0$ and $q_{i j^{\prime}}>0$ implies $\frac{p_{j}}{c_{i j}}=\frac{p_{j^{\prime}}}{c_{i j^{\prime}}}=\min _{k \in \mathcal{J}} \frac{p_{k}}{c_{i k}}$. In particular, $q_{i j}>0$ and $q_{i j^{\prime}}>0$ implies

$$
\frac{c_{i j}}{c_{i j^{\prime}}}=\frac{p_{j}}{p_{j^{\prime}}} .
$$

We now consider the BGR defined by the support sets $\left\{\hat{\mathcal{J}}_{i}\right\}_{i=1}^{I}$ of undecided users. For any two edges $(i, j)$ and $(i, k)$ of BGR, where $i$ is a user index and $j, k$ are provider indices, $q_{i j}>0$ and $q_{i k}>0$ so by (14) we have $\frac{c_{i j}}{c_{i k}}=\frac{p_{j}}{p_{k}}$.

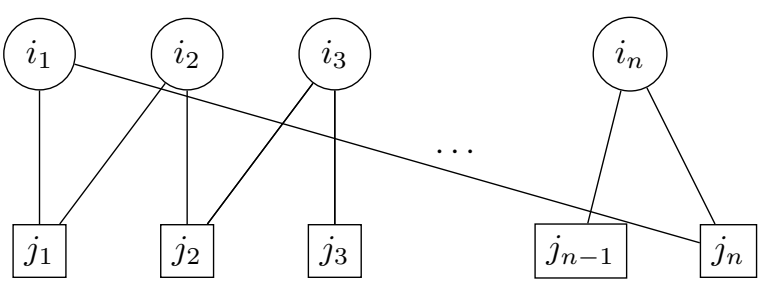

Fig. 3. A bipartite graph representation loop

Suppose that a loop exists in BGR (refer to Figure 3 for this part of the proof). Then, a sequence of nodes $i_{1}, j_{1}, i_{2}, j_{2}, \ldots$, $i_{n}, j_{n}, i_{1}$ exists, where $i_{1}, \ldots, i_{n}$ are the user nodes and $j_{1}, \ldots, j_{n}$ are the provider nodes, such that $\left(i_{k}, j_{k}\right)$ and $\left(j_{k-1}, i_{k}\right)$ are edges in BGR for $k=1, \ldots n$ (with $0=n$ ). The members of the sequence are distinct (if they are not, that only means that there is a smaller loop that we can consider). Since both $\left(i_{k}, j_{k}\right)$ and $\left(j_{k-1}, i_{k}\right)$ are edges, equation (14) implies that $\alpha_{i_{k}}^{j_{k-1} j_{k}}=\frac{c_{i k-1}}{c_{i k}}=\frac{p_{k-1}^{*}}{p_{k}^{*}}$. Hence, $\alpha_{i_{1}}^{j_{n} j_{1}}=\frac{p_{n}^{*}}{p_{1}^{*}}$, $\alpha_{i_{2}}^{j_{1} j_{2}}=\frac{p_{1}^{*}}{p_{2}^{*}}, \ldots, \alpha_{i_{n-1}}^{j_{n-2} j_{n-1}}=\frac{p_{n-2}^{*}}{p_{n-1}^{*}}, \alpha_{i_{n}}^{j_{n-1} j_{n}}=\frac{p_{n-1}^{*}}{p_{n}^{*}}$. From this, it can be concluded that a loop in the preference graph implies:

$\alpha_{i_{1}}^{j_{n} j_{1}} \alpha_{i_{2}}^{j_{1} j_{2}} \ldots \alpha_{i_{n-1}}^{j_{n-2} j_{n-1}} \alpha_{i_{n}}^{j_{n-1} j_{n}}=\frac{p_{n}^{*}}{p_{1}^{*}} \frac{p_{1}^{*}}{p_{2}^{*}} \ldots \frac{p_{n-2}^{*}}{p_{n-1}^{*}} \frac{p_{n-1}^{*}}{p_{n}^{*}}=1$.

Note that $\alpha_{i}^{j j^{\prime}}=\frac{c_{i j}}{c_{i j^{\prime}}}$ is the ratio of two independent continuous random variables, hence it is a continuous random variable itself, for any $i \in \mathcal{I}, j, j^{\prime} \in \mathcal{J}$. Also $\alpha_{i}^{j j^{\prime}}$ is independent from $\alpha_{l}^{k k^{\prime}}$ for $l \neq i \in \mathcal{I}$ and all $j, j^{\prime}, k, k^{\prime} \in \mathcal{J}$. However the probability that the product of independent continuous random variables equals a constant is zero. In other words, 
the event that a BGR has a loop is of zero measure.

\section{APPENDIX B \\ PROOF OF THEOREM 4}

Assume that the providers charge prices $\boldsymbol{p}=\left[p_{1} \ldots p_{J}\right]$ to the users. Then, each user faces a local maximization problem $\mathrm{USER}_{i}(\boldsymbol{p})$ :

$$
\begin{aligned}
& \max u_{i}\left(\sum_{j=1}^{J} q_{i j} c_{i j}\right)-\sum_{j=1}^{J} p_{j} q_{i j} \\
& \text { over } q_{i j} \geq 0, \forall j \in \mathcal{J}
\end{aligned}
$$

This is a non-strictly concave maximization problem. By differentiating and looking at the first order conditions, we conclude that the maximizing conditions are:

$$
\begin{aligned}
\frac{\partial u_{i}\left(\sum_{j=1}^{J} q_{i j} c_{i j}\right)}{\partial q_{i j}}=\frac{\partial u_{i}\left(x_{i}\right)}{\partial x_{i}} c_{i j}-p_{j} & =0, q_{i j}>0 \\
& \leq 0, q_{i j}=0
\end{aligned}
$$

where $x_{i}=\sum_{j=1}^{J} q_{i j} c_{i j}$. Since $u_{i}(\cdot)$ is strictly increasing and strictly concave, there is a unique value of $x_{i}$ that is maximizing a user's payoff. Equations (16) and (17), together with $x_{i}=\sum_{j=1}^{J} q_{i j} c_{i j}$ are equivalent to equations (9)-(11) and (13). Furthermore, for a demand vector to be a subgame perfect equilibrium of the provider competition game, it is required that the demand each provider is facing equals to the supply, i.e. $\sum_{i=1}^{I} q_{i j}=Q_{j}$, for all $j \in \mathcal{J}$, which is equation (12). Hence, the SPE is a price vector tuple $\boldsymbol{p}, \boldsymbol{q}$ that satisfies KKT conditions (9)-(13). But, any vector tuple $\boldsymbol{p}, \boldsymbol{q}$ that satisfies the KKT conditions is a maximizing solution of NUM. Furthermore, we know that there is exactly one such tuple. Hence, we have established formal equivalence between the SPE of the provider competition game and the maximizing demand vector of NUM.

\section{REFERENCES}

[1] P. Marbach, "Analysis of a static pricing scheme for priority services," IEEE/ACM Trans. on Networking, vol. 12, no. 2, pp. 312-325, 2004.

[2] J. Huang, R. A. Berry, and M. L. Honig, "Auction-based spectrum sharing," Mob. Netw. Appl., vol. 11, no. 3, pp. 405-418, 2006.

[3] F. Kelly, A. Maulloo, and D. Tan, "Rate control for communication networks: Shadow prices, proportional fairness and stability," Journal of the Operational Research Society, vol. 49, pp. 237-252, 1998.

[4] T. Basar and R. Srikant, "Revenue-maximizing pricing and capacity expansion in a many-users regime," INFOCOM 2002 Proceedings, vol. 1, pp. 294-301 vol.1, 05 2002/05/31.

[5] H. Shen and T. Basar, "Differentiated internet pricing using a hierarchical network game model," American Control Conference, 2004. Proceedings of the 2004, vol. 3, pp. 2322-2327 vol.3, 01 2004/01/01.

[6] P. Maille and B. Tuffin, "Analysis of price competition in a slotted resource allocation game," in INFOCOM 2008. The 27th Conference on Computer Communications. IEEE, 2008, pp. 888-896.

[7] U. Varshney and R. Vetter, "Mobile commerce: Framework, applications and networking support," Mobile Networks and Applications, vol. 7, no. 3, pp. 185-198, 06 2002/06/01/.

[8] L. Buttyán and J.-P. Hubaux, "Stimulating cooperation in self-organizing mobile ad hoc networks," Mob. Netw. Appl., vol. 8, no. 5, pp. 579-592, 2003.

[9] C. U. Saraydar, N. B. Mandayam, and D. J. Goodman, "Efficient power control via princing in wireless data networks," IEEE Transactions on Communications, vol. 50, no. 2, pp. 291-303, February 2002.
[10] P. Marbach and R. Berry, "Downlink resource allocation and pricing for wireless networks," vol. 3, 2002, pp. 1470-1479 vol.3.

[11] M. Chiang and J. Bell, "Balancing supply and demand of bandwidth in wireless cellular networks: Utility maximization over powers and rates," in Proceedings of IEEE INFOCOM, vol. 4, 2004, pp. 2800-2811.

[12] D. Acemoglu, A. Ozdaglar, and R. Srikant, "The marginal user principle for resource allocation in wireless networks," in 43rd IEEE Conference on Decision and Control, vol. 2, 2004, pp. 1544-1549 Vol.2.

[13] J. Musacchio and J. Walrand, "Wifi access point pricing as a dynamic game," IEEE/ACM Transactions on Networking, vol. 14, no. 2, 04 2006/04/01

[14] S. Adlakha, R. Johari, and A. J. Goldsmith, "Competition in wireless systems via bayesian interference games," CoRR, vol. abs/0709.0516, 2007, informal publication.

[15] R. Etkin, A. Parekh, and D. Tse, "Spectrum sharing for unlicensed bands," in DySPAN, 2005, pp. 251-258.

[16] J. Huang, R. A. Berry, and M. L. Honig, "Distributed interference compensation for wireless networks," Selected Areas in Communications, IEEE Journal on, vol. 24, no. 5, pp. 1074-1084, 2006.

[17] C. Zhou, M. L. Honig, and S. Jordan, "Utility-based power control for a two-cell CDMA data network," Wireless Communications, IEEE Transactions on, vol. 4, no. 6, pp. 2764-2776, 2005.

[18] L. Grokop and D. N. Tse, "Spectrum sharing between wireless networks," in Proceedings of IEEE INFOCOM, 2008.

[19] A. Zemlianov and G. de Veciana, "Cooperation and decision-making in a wireless multi-provider setting," in Proceedings of IEEE INFOCOM 2005, vol. 1, 2005, pp. 386-397 vol. 1.

[20] S. Sengupta, M. Chatterjee, and S. Ganguly, "An economic framework for spectrum allocation and service pricing with competitive wireless service providers," in DySPAN, 2007, pp. 89-98.

[21] J. Jia and Q. Zhang, "Competitions and dynamics of duopoly wireless service providers in dyn. spectrum market," in MobiHoc, 2008.

[22] O. Ileri, D. Samardzija, T. Sizer, and N. B. Mandayam, "Demand responsive pricing and competitive spectrum allocation via a spectrum server," in DySPAN, 2005, pp. 194-202.

[23] J. Acharya and R. D. Yates, "Service provider competition and pricing for dynamic spectrum allocation," in International Conference on Game Theory for Networks (GameNets), 2009.

[24] D. Acemoglu and A. Ozdaglar, "Price competition in communication networks," in INFOCOM 2006. 25th IEEE International Conference on Computer Communications. Proceedings, 2006, pp. 1-12.

[25] — - "Competition and efficiency in congested markets," Math. Oper. Res., vol. 32, no. 1, pp. 1-31, 2007.

[26] H. Inaltekin, T. Wexler, and S. B. Wicker, "A duopoly pricing game for wireless ip services," in SECON, 2007, pp. 600-609.

[27] S. Leyffer and T. Munson, "Solving multi-leader-follower games," Preprint ANL/MCS-P1243-0405, 04 2005/04/22.

[28] J. Wang, D. Chiu, and J. Lui, "A game-theoretic analysis of the implications of overlay network traffic on isp peering," Computer Networks, vol. 52, no. 15, pp. 2961-2974, 10 2008/10/23.

[29] X. Lin and N. B. Shroff, "Utility maximization for communication networks with multipath routing," Automatic Control, IEEE Transactions on, vol. 51, no. 5, pp. 766-781, 2006.

[30] T. Voice, "Stability of congestion control algorithms with multi-path routing and linear stochastic modelling of congestion control," Ph.D. dissertation, University of Cambridge, Cambridge, UK, May 2006.

[31] V. Gajic, J. Huang, and B. Rimoldi, "Competition of wireless providers for atomic users: Equilibrium and social optimality," Technical report, 2009. [Online]. Available: http://personal.ie.cuhk.edu.hk/ jwhuang/ publication/comp_tech.pdf

[32] D. P. Bertsekas, Nonlinear Programming. Athena Scientific, Sept. 1999.

[33] D. Fudenberg and J. Tirole, Game Theory. MIT Press, 1991.

[34] V. Gajic, J. Huang, and B. Rimoldi, "Competition of wireless providers for atomic users: Distributed algorithm design," Working paper, 2009.

[35] J. Mo and J. Walrand, "Fair end-to-end window-based congestion control," Networking, IEEE/ACM Transactions on, vol. 8, no. 5, pp. 556-567, 10 2000/10/01.

[36] A. Rad, J. Huang, M. Chiang, and V. Wong, "Utility-optimal random access without message passing," Wireless Communications, IEEE Transactions on, vol. 8, no. 3, pp. 1073-1079, 2009.

[37] D. Wong and T. J. Lim;, "Soft handoffs in cdma mobile systems," Personal Communications, IEEE [see also IEEE Wireless Communications], vol. 4, no. 6, pp. 6-17, 12 1997/12/01. 